\title{
Inicio de las transiciones a la adultez de jóvenes de segunda generación de origen mexicano en el sur de California
}

\section{Beginning of Transitions to Adulthood for Second Generation Youth of Mexican Origin in Southern California}

\author{
María del Rosario Narváez Jiménez \\ U. Autónoma de Morelos, México \\ mariarosarionarvaez@gmail.com
}

\begin{abstract}
Resumen: El objetivo de este artículo es conocer cómo inician los jóvenes de segunda generación de origen mexicano sus transiciones a la adultez, tomando como ejes de análisis las trayectorias educativas en sus comienzos y partiendo de dos instituciones sociales como sus principales influencias, la familia y la escuela. El enfoque biográfico, concretamente los relatos de vida desde la perspectiva etnosociológica de Daniel Bertaux, ha sido el encuadre desde el que se ha llevado a cabo el análisis y la interpretación de los datos. El trabajo de campo se ha desarrollado en el sur del estado de California, en 2016 y durante un periodo de seis meses. Los jóvenes entrevistados fueron 51, pero en este trabajo se utilizarán los relatos de siete de los informantes.
\end{abstract}

Palabras claves: transición a la adultez, joven, segunda generación, mexicano.

Abstract: The aim of this article is to find out how second generation young people of Mexican origin begin their transitions to adulthood, taking as the main axes of analysis the educational trajectories in their beginnings and starting from two social institutions as their main influences, the family and the school. The biographical approach, specifically Daniel Bertaux's life stories from an ethno-sociological perspective, has been the framework from which the analysis and interpretation of the data has been carried out. The field work was carried out in the south of the state of California, in 2016 and over a period of six months. Fifty-one young people were interviewed, but this study will use the accounts of seven of the informants.

Keywords: transition to adulthood, young, second generation, mexican. 


\section{INTRODUCCIÓN: DE LA TEORÍA DE LA ASIMILACIÓN AL ENFOQUE DE LAS TRANSICIONES A LA ADULTEZ}

La teoría de la asimilación ha sido la perspectiva dominante en las investigaciones en materia de inmigración desde principios de siglo xx en Estados Unidos de América (EUA). Los primeros inmigrantes en llegar procedían de países angloculturales y protestantes, por ello, cuando al principio del siglo xx comenzó a llegar la inmigración procedente de los países mediterráneos y eslavos (Giner et al., 1998), las diferencias culturales, religiosas y lingüísticas se convirtieron en un problema debido a sus dificultades de asimilación. De igual manera, en el ámbito europeo, aunque con diferentes perspectivas e intereses, el objetivo ha sido parecido. Las investigaciones han respondido al interés por conocer los resultados de las diferentes políticas de integración dirigidas a las segundas generaciones. Y estas han mostrado las consecuencias en función del enfoque político: el asimilacionista francés, el multiculturalista de Reino Unido o el de comunidad étnica de Alemania (Aparicio, 2007).

Por tanto, la perspectiva de la asimilación, así como sus variantes, y las investigaciones en el contexto europeo han compartido un objetivo común, conocer cómo se ha llevado a cabo la integración de las segundas generaciones. Otro de los aspectos habituales ha sido tomar el elemento cultural como característica definitoria de las segundas generaciones; mientras que los componentes socioestructurales no han sido tenidos en cuenta o han sido relegados a un segundo plano. Por ello, los diferentes modelos de integración tienen una perspectiva unilateral de la asimilación y no dan cuenta del contexto más amplio en el que se producen ni del proceso. Pero hay otro elemento que también ha sido obviado, la etapa de la vida en la que se encuentran los miembros de la segunda generación, la juventud, asumiendo que las implicaciones de pertenencia a dicha categoría van más allá de la edad.

Por otro lado, el componente étnico de la segunda generación no solamente debe incluir aspectos culturales, sino también socioestructurales. De esta manera, la concepción de segunda generación ha quedado integrada por dos dimensiones, la juventud concebida como proceso de transición a la adultez y la etnicidad convertida en eje estructurador de las relaciones sociales que determinará el resto de estatus y roles de los jóvenes de origen mexicano (Epstein, 2006), donde se incluyen el estatus socioeconómico y el género. Por tanto, desde el enfoque de las transiciones a la adultez se propone un planteamiento diferente basado en los procesos de inserción social de los jóvenes de segunda generación; en lugar de los procesos de integración social analizados desde las diferentes propuestas asimilacionistas. $Y$ donde los aspectos socioestructurales, como la 
situación social de los jóvenes y de sus familias en función de su condición étnica ocuparán un lugar central en el análisis.

De esta manera, la perspectiva de las transiciones a la adultez y la concepción de segunda generación en base al origen étnico constituyen un nuevo enfoque en el estudio de las segundas generaciones. De ahí que el objetivo de este artículo sea conocer cómo inician los jóvenes de segunda generación de origen mexicano sus transiciones a la adultez, tomando como ejes de análisis las trayectorias educativas en sus comienzos y partiendo de dos instituciones sociales como sus principales influencias, la familia y la escuela. El enfoque biográfico, concretamente los relatos de vida desde la perspectiva etnosociológica de Daniel Bertaux (2005), ha sido el encuadre desde el que se ha llevado a cabo el análisis y la interpretación de los datos. El trabajo de campo se ha desarrollado en el sur del estado de California, en 2016 y durante un periodo de seis meses. Los jóvenes entrevistados fueron 51, pero en este trabajo se utilizarán los relatos de siete de los informantes.

\section{LA JUVENTUD COMO TRANSICIÓN A LA ADULTEZ}

Para Bourdieu (2002), la juventud es un concepto que remite a una construcción social, una categoría social compuesta en base a unos supuestos, donde lo primordial no reside en los elementos que la integran, sino en su trama de relaciones. De esta manera el establecimiento de la categoría joven implica la constitución de posiciones sociales de un sistema de relaciones sociales que se reproducen en base a la lucha por la distribución de poderes entre los sujetos que ocupan dichas posiciones. Por tanto, las diferencias entre jóvenes y adultos irán aparejadas a un conjunto de estatus y roles sociales asociados a cada categoría (Benedict, 2008). Posiciones sociales que corresponderán a las etapas en que se divide el curso de vida: infancia, juventud, madurez y vejez. Dentro de este sistema de relaciones, el poder está acaparado por los adultos frente a los jóvenes, con los que se establece una lucha por el poder.

Por consiguiente, la juventud será entendida como transición a la adultez y con características particulares entre los diversos contextos culturales (Benedict, 2008). En lo que respecta a las sociedades occidentales, Galland (1991) define la transición a la vida adulta como el periodo en que se finalizan los estudios y se produce la entrada en el mercado de trabajo. De esta manera, se alcanza la independencia económica, al mismo tiempo que se abandona la familia de origen y se constituye un nuevo hogar y una nueva familia. Así, tiene lugar el modelo de transición lineal a la vida adulta. Pero este modelo ha dejado de tener vigencia. Los cambios sociales y económicos que empiezan a produ- 
cirse a partir de la segunda mitad del siglo xx traen consigo una situación de mayor inestabilidad y riesgos que, a su vez, desencadenan desestandarización y discontinuidad en las trayectorias familiares y profesionales (Cavalli, 2007). Las estructuras de transición se vuelven bifurcadas, reversibles e individuales (Dávila y Ghiardo, 2011; Gil Calvo, 2001; Pais, 2007). En definitiva, los caminos para convertirse en adulto son cada vez más variados, estando en función de los contextos sociohistóricos y culturales, así como de las circunstancias personales de los individuos; es decir, en función del estatus socioeconómico, el género y la etnia (Dávila y Ghiardo, 2011).

\section{DEL MODELO LINEAL A LA HETEROGENEIDAD: CARACTERÍSTICAS DE LAS TRANSICIONES ACTUALES}

Las sociedades actuales se caracterizan por el cambio continuo, de manera que las transformaciones que comienzan en lo social acaban impactando en las biografías personales (Gil Calvo, 2001), que se vuelven más individualizadas y desestandarizadas, ya que las transiciones carecen de estructuras, de modelos prefijados que dirijan su curso. Así, se pasa de un modelo de transición lineal, aún hoy vigente, a modelos más complejos y heterogéneos (Singly, 2005). Por tanto, si hay algo que caracteriza a los modelos de transición actuales, es que se desinstitucionalizan, o se reinstitucionalizan (Pais, 2007), y se vuelven más diferenciados e individualizados (Mayer, 2001). Estas características serán entendidas por Moreno Mínguez et al. (2012) de la siguiente manera: la desinstitucionalización está referida al proceso mediante el cual los itinerarios se hacen más flexibles, lo que a su vez conlleva mayor reversibilidad y discontinuidad; la diferenciación conlleva la diversificación de los itinerarios como consecuencia de factores estructurales (inestabilidad laboral, diversificación y alargamiento de la etapa educativa) y de nuevas pautas sociales (nuevas formas familiares, relaciones personales, etc.); y la individualización coincide con los cambios en la condición juvenil que origina situaciones de mayor control sobre sus decisiones.

La consecuencia de todos estos cambios es el fin del modelo lineal de transición y el surgimiento de nuevos modelos, más heterogéneos y fragmentarios. De esta manera, los jóvenes se ven obligados a hacer frente a transiciones que no cuentan con una estructura fija, por lo que se verán más expuestos a eventos inesperados y a una mayor incertidumbre a la hora de trazar sus itinerarios futuros. Por tanto, las consecuencias ambivalentes de la desestandarización e individualización de las transiciones conllevan que las transformaciones, que tienen su origen en lo social, se tornen cada vez más personales y se conviertan, además de en riesgos e incertidumbre, en nuevas posibilidades. Pues el 
debilitamiento de las instituciones y un mayor grado de individualización permiten a los individuos convertirse en actores, agentes de proyectos biográficos propios (Cavalli, 2007). Los nuevos modelos presentan mayores probabilidades de bifurcación y reversibilidad, lo que puede ser interpretado como una menor seguridad a la hora de emprender proyectos futuros, pero también representan nuevas oportunidades para volver a empezar. Así, la individualización de las biografías (Beck y Beck-Gernsheim, 2003) implica autonomía y elección, pero se siguen manteniendo las diferencias de clases, género y etnia.

\section{FAMILIA Y ESCUELA}

En los procesos de transición a la adultez hay que destacar dos instituciones sociales que constituyen las bases de la socialización juvenil, al mismo tiempo que son el origen de las relaciones de dominación de los adultos sobre los jóvenes; estas son la familia y la escuela (Parsons, 2008). Las relaciones más básicas de la organización social son las que se producen en base a las edades heterogéneas y se originan dentro del grupo familiar. Se basan en la interacción asimétrica de poder y autoridad que ejercen los adultos sobre los niños (Eisenstadt, 2008). Por consiguiente, las relaciones entre jóvenes y adultos se originan en el seno de la familia, pero estas dependerán de aspectos sociales y culturales. En las sociedades occidentales, la familia nuclear ${ }^{1}$ constituye la base de dichas relaciones.

El surgimiento de la familia nuclear tiene lugar tras la revolución industrial, como había sido sugerido por Burgess en 1916, años más tarde por Ogburn y también por Parsons (Bengston, 2001). Sin embargo, desde la década de los 60 del siglo pasado se produce una serie de cambios que repercuten en las estructuras y funciones de las familias, que Poponoe interpreta como el declive de la familia nuclear, mientras que otros relacionan con una mayor heterogeneidad (Bengston, 2001). Pero dejando de lado las disputas, lo acontecido fue una serie de cambios que alteraron las relaciones intergeneracionales, entre otros aspectos. Así, surgió un nuevo tipo de familia, la «familia negociadora», que se caracteriza por el establecimiento de relaciones más democráticas e igualitarias (Meil, 2011).

${ }^{1}$ La definición del término familia es muy compleja debido a su diversidad. Se trata de una institución universal que «designa a un grupo social constituido por personas vinculadas por la sangre, el matrimonio o la adopción, caracterizado por una residencia común, cooperación económica, reproducción y cuidado de la descendencia» (Giner et at., 1998: 294). 
Las nuevas relaciones establecidas entre los jóvenes y sus familias de origen son consecuencia de algunos de los cambios producidos en las transiciones a la adultez. Aunque la familia continúa manteniendo sus funciones tradicionales de refugio, intimidad y servicios materiales ante la inestabilidad y precariedad del mercado de trabajo (Esping-Andersen, 2000), también adquiere nuevas funciones, como el apoyo a los procesos de individualización de los hijos (Walter, 2004). Por tanto, se trata de un cambio en las relaciones intergeneracionales que pasan del conflicto a la reestructuración (Leccardi, 2010), mediante un pacto de solidaridad entre padres e hijos. Pacto que será consecuencia de la transformación y prolongación de la juventud (Cavalli y Galland, 1995), pero también de la imprevisibilidad y el riesgo generado por las nuevas condiciones. Ello conduce a una mayor dependencia de los jóvenes respecto de sus familias, situación que «obliga» a ambas generaciones a una redefinición de sus relaciones. En este sentido, Beck y Beck-Gernsheim (2003) hacen referencia a la «familia post-familiar» como consecuencia de las nuevas relaciones surgidas de las situaciones adversas, que suponen el paso de relaciones tradicionales sustentadas en lo material a otras que lo hacen en la solidaridad. Por tanto, en el ámbito familiar se siguen manteniendo las relaciones tradicionales sustentadas en la base material, al mismo tiempo que surgen nuevas relaciones menos asimétricas y donde los vínculos intergeneracionales adquieren mayor protagonismo. Esto indica que la familia se encuentra en plena transición, lo que supone una redefinición de los roles de sus miembros en cuanto a las relaciones de género y a las relaciones entre jóvenes y adultos (Meil, 2006; 2011).

La escuela será la otra institución de referencia al hablar de juventud. Así la concibe Reguillo (2012), para quien el concepto de juventud debe incluir todos aquellos aspectos que como categoría social la integran. Para ello será necesario aprehender sus fronteras y los contenidos que en ella se incluyen; por lo que habrá de incorporar el contexto y los actores que componen el sistema, entre los que se incluyen el Estado y la escuela. Así como la concepción de los jóvenes sobre su propia situación, donde el presente sustituye a la concepción futura que de ellos se tiene desde el mundo adulto. De igual manera, Carles Feixa (1998) afirma que un análisis de la juventud debe incluir su interrelación con otros componentes sociales, ya que es esta la única forma en la que adquiere sentido. Por tanto, la escuela como institución socializadora por excelencia, además de la familia, supone la escolarización masiva de la infancia desde finales del siglo xix (Giner et al., 1998). Ello es resultado de la institucionalización de la educación que conlleva la separación formalizada, primero del niño y más tarde del joven, del mundo adulto (Musgrove, 2008).

En el mismo sentido, Saraví (2015) concibe la escuela como la principal institución relacionada con la juventud. De esta manera, «la experiencia y el sentido mismo de la 
juventud» ha cambiado acorde a las transformaciones del sistema educativo formal; al mismo tiempo que ha supuesto su institucionalización como «etapa del curso de vida». Pero también se encarga de señalar que otra de las funciones de la escuela, además del «proceso de aprendizaje y transmisión de conocimientos entre generaciones», es la de «espacio por excelencia de construcción de ciudadanía o pertenencia» (Saraví, 2015: 82).

De esta manera, las relaciones de autoridad y poder ejercidas por los adultos sobre los jóvenes se extienden de la familia a la escuela. Lo que conlleva que la institución educativa acabe favoreciendo la asociación de los niños y jóvenes con sus pares. A través del grupo de pares los jóvenes establecen relaciones de vital importancia con sus coetáneos, que acabarán convirtiéndose en grupo de referencia para los mismos. Por tanto, en función de la vinculación que se da entre juventud y escuela se hará especial hincapié en esta como institución que posibilita las relaciones entre niños y jóvenes y sus pares de diferentes orígenes. Así, el proceso de construcción de pertenencia por parte de los jóvenes de origen mexicano (Suárez-Orozco y Suárez-Orozco, 2003; Portes y Rumbaut, 2010; Saraví, 2015) tendrá la escuela como escenario primordial, ya que se trata de la principal institución asimiladora en las etapas de la niñez y de la juventud.

\section{ENFOQUE BIOGRÁFICO: RELATOS DE VIDA}

El enfoque biográfico, concretamente los relatos de vida desde la perspectiva etnosociológica de Daniel Bertaux (2005), ha sido el encuadre desde el que se ha llevado a cabo el análisis y la interpretación de los datos. Bertaux (1999) parte de la diferenciación que en lengua inglesa se hace entre los términos life story, relato de vida, y life history, historia de vida. Toma como referencia la propuesta de Norman K. Denzin, quien basándose en esta distinción comprende cada uno de estos términos de la siguiente manera: life story, o relatos de vida, se refiere a «la historia de una vida tal como la cuenta la persona que la ha vivido» (Bertaux, 1999: 3), mientras que life history, o historia de vida, comprende «los estudios de casos sobre una persona [...] incluyendo no sólo su propio relato de vida, sino también otras clases de documentos» como «la historia clínica, el expediente judicial [...] los testimonios de allegados, etc.» (Bertaux, 1999: 3). Y se posiciona a favor de la opción de enfoque biográfico, pues de esta manera se asume «la adopción de una nueva técnica», pero también «la construcción paulatina de un nuevo proceso sociológico» (Bertaux, 1999: 3), «de un nuevo enfoque» donde se concilian «la observación y la reflexión» (Bertaux, 1999: 4).

Para la perspectiva etnosociológica, los relatos de vida son entendidos como una de las formas de expresión de la experiencia humana (Bertaux, 2005). Se «considera al 
interlocutor como un informante» (Bertaux, 1999: 10) y al relato, la narración que una persona hace de su propia experiencia de vida. Se trata de una autobiografía, ya que es la persona quien narra su vida. Así, el relato tiene lugar a través del diálogo que se produce entre el sujeto y el investigador; por lo que se produce a petición del investigador, quien tendrá unos intereses de conocimientos particulares sobre dicha experiencia, limitándose a uno o varios ámbitos del todo de esa experiencia de vida.

El objetivo central del relato de vida será reflejar la estructura diacrónica de la experiencia vital a través de la narración de parte de la experiencia vivida. De esta manera, la entrevista se ha enfocado desde el propio curso de vida con el propósito de poder conocer cómo se ha desarrollado la experiencia vital del joven, desde la infancia, pasando por el comienzo de la juventud y su evolución durante esta etapa hasta la actualidad. Por tanto, aunque la perspectiva etnosociológica se inspira en la tradición etnográfica, sus objetivos se construyen en base a un matiz sociológico. Esto es, «un tipo de investigación empírica basada en el trabajo de campo, inspirado en la tradición etnográfica para sus técnicas de observación, pero que construye sus objetivos por referencia a ciertas problemáticas sociológicas» (Bertaux, 2005: 15). Mientras que la etnografía será entendida como un modo de hacer interpretación de lo que otros cuentan y de las propias observaciones del investigador. Serán interpretaciones de interpretaciones, interpretaciones «de segundo y tercer orden» (Geertz, 1973: 28) y concebidas como ficciones por tratarse de algo que ya está hecho, pero que no hay que confundir con lo ficticio.

Siguiendo el procedimiento de la perspectiva etnosociológica, se ha partido del análisis e interpretación interna de los relatos, así como de la comparación de casos variados, con el objetivo de generalizar, de realizar una transición desde lo particular a lo general, procedimiento característico del modelo inductivo. El procedimiento ha consistido en la comparación de diversos relatos de vida, con el fin de elaborar conceptos y categorías como resultado del proceso de interpretación. Así, cada relato ha sido interpretado como parte del conjunto que suponen los relatos vida de los jóvenes de segunda generación de origen mexicano en el sur del estado de California. De esta manera, los conceptos y categorías resultantes han ayudado a la elaboración de «hipótesis plausibles».

Esta forma de proceder se distancia del modelo de investigación hipotético deductivo, que se caracteriza por partir de lo general para desembocar en lo particular, se desarrolla mediante la verificación de hipótesis y cuyo objetivo es la explicación; ello, a su vez, repercute en la construcción de la muestra, ya que no se trata de una muestra estadísticamente representativa, sino que se ha construido de manera progresiva, a la par de la recogida de datos y de la búsqueda de semejanzas y diferencias entre los distintos casos a comparar. Este es el procedimiento de la Teoría Fundamentada de Glasser y 
Strauss, cuya propuesta reside en el método de la comparación constante y en el muestreo teórico, así como en la obtención de los datos que proceden de la realidad observada y registrada.

\section{CARACTERÍSTICAS DE LA MUESTRA Y PROCESO DE CATEGORIZACIÓN DE LAS TRANSICIONES A LA ADULTEZ}

Con el fin de delimitar la población integrante de la segunda generación se ha tomado como referencia la propuesta de Rubén G. Rumbaut (2006), quien diferencia entre «primera generación ${ }^{2} »$, integrada por los nacidos en el extranjero y que subdivide en las generaciones: $1.75,1.5$ y 1.25, en función de la edad de llegada al nuevo país. Y la «segunda generación ${ }^{3} \gg$, que incluye a los nacidos en EUA e integra a las generaciones 2.5 y 2.0. En este trabajo se considerará a los jóvenes nacidos en EUA y a los nacidos en el extranjero integrantes de la segunda generación, ya que muchos de estos últimos, debido a la temprana edad a la que llegaron a EUA, han tenido un proceso de socialización similar al de los nativos.

El trabajo de campo se ha desarrollado en el sur del estado de California, concretamente en los condados de Orange y Los Ángeles, los más poblados y con una importante proporción de población de origen mexicano; durante un periodo de seis meses, comprendido entre marzo y agosto de 2016. Los jóvenes entrevistados fueron 51; de ellos 24 son mujeres y 27 son hombres, con edades comprendidas entre los 16 y 36 años. En cuanto a la generación, 32 jóvenes pertenecen a la generación 2.0, es decir, nacieron en EUA, la mayoría en el estado de California; mientras que los 19 restantes son migrantes, de los que 10 pertenecen a la generación $1.75 ; 5$, a la generación $1.5 ; \mathrm{y} 4$, a la generación 1.25. Y en cuanto a sus estatus legales, 11 cuentan con residencia permanente o ciudadanía, mientras que de los otros 13, 7 se encuentran bajo la protección de DACA y 6 están indocumentados.

El proceso de transición a la adultez ha sido dividido en diferentes fases, cada una de ellas referida al momento de la transición en el que se encontraban los jóvenes en

${ }^{2}$ La generación 1.75 incluye a los jóvenes que llegaron a EUA en el intervalo de edad comprendido entre 0 y 5 años. La generación 1.5 se refiere a los que llegaron cuando se encontraban entre los 6 y 12 años de edad. Y la generación 1.25 está integrada por los que llegaron cuando tenían entre 13 y 17 años.

${ }^{3}$ La generación 2.5 se refiere a los nacidos en EUA, de un padre nativo estadounidense y otro nacido en el extranjero; y la generación 2.0 incluye a los nacidos en EUA, pero de ambos padres nacidos en el extranjero. 
relación con los eventos clásicos que la definen (finalización de los estudios, incorporación al mercado laboral y formación de una nueva familia). Para ello se tomaron como ejes de análisis las distintas trayectorias (educativas, laborales y familiares) y las situaciones respecto a las mismas en las que se encontraban los informantes al momento de la entrevista. Así, las fases de las transiciones constituyen las categorías principales del proceso, que son: «fase inicial», «fase intermedia» y «fase avanzada»; las cuales han sido divididas, a su vez, en diversas subcategorías en base al rol principal desempeñado por los jóvenes en el momento de la entrevista.

Este trabajo se centrará en la primera categoría, «fase inicial», que marca el inicio de las transiciones a la adultez y corresponde a jóvenes que se encuentran finalizando la preparatoria; iniciando o terminando sus trayectorias educativas superiores; e iniciando la trayectoria laboral, entre los que no han continuado estudiando después de la preparatoria. Casi todos ellos residen con sus padres, y en los casos en que no lo hacen se debe a motivos educativos, por lo que su independencia residencial se considera temporal. Debido a la situación en la que se encuentran, estudiando o iniciando la trayectoria laboral, no disponen de independencia económica, por lo que son los jóvenes con mayor dependencia material de sus progenitores; y sus edades oscilan entre los 17 y los 24 años, por lo que se trata de los de menor edad de la muestra.

A continuación se expondrán algunos de los casos pertenecientes a la «fase inicial», concretamente, los fragmentos de relatos ${ }^{4}$ de vida de siete de los informantes. Se trata de jóvenes que están finalizando la preparatoria o han continuado sus trayectorias educativas en colegios comunitarios o universidades como actividad principal, aunque algunos de ellos también realizan alguna actividad laboral complementaria, pero de manera secundaria; casi todos residen en el hogar familiar, a excepción de dos jóvenes, cuyas salidas de la residencia familiar se deben a motivos educativos. Con respecto a los aspectos a los que los informantes han hecho referencia en sus relatos, estos son múltiples y complejos, pero aquí solo se incluirán los relacionados con las trayectorias educativas por constituir el eje de análisis en este trabajo. Destacan la escuela, como contexto donde tienen lugar gran parte de las experiencias que influyen en los itinerarios de transición; las relaciones intergeneracionales, concretamente las relaciones paternofiliales en función de las diferentes aspiraciones y expectativas sobre los proyectos de vida de los jóvenes; la cohorte de pertenencia y el estatus legal, por la condición de ciudadanía, o no, y/o el momento en que se produce el evento migratorio, por las dificultades y o fa-

${ }^{4}$ La transcripción de los relatos se ha realizado de manera literal con el fin de plasmar de la manera más realista posible la forma en que se produjeron los discursos. 
cilidades que ambos aspectos puedan suponer; así como las situaciones y experiencias particulares de los informantes.

\section{LOS JÓVENES FRENTE A LOS ÁMBITOS ADULTOS: FAMILIA Y ESCUELA}

Las relaciones de autoridad ejercida por los adultos sobre los jóvenes tienen su máxima expresión en dos instituciones sociales, la familia y la escuela; por ello, han sido estos los dos ámbitos sobre los que han girado los relatos. Para Benedict (2008), uno de los contrastes en cuanto a los roles de niños y adultos es la relación entre el dominio y la sumisión. Por tanto, desde las relaciones establecidas en el seno familiar, las paternofiliales han sido uno de los elementos centrales de los relatos. En segundo lugar, la escuela constituye la principal institución a través de la cual los niños comienzan a tomar contacto con la sociedad más allá de sus familias, y cuya función socializadora se basa en la transmisión de las normas y valores socioculturales. En este sentido, es aquí donde los niños adquieren y perfeccionan el idioma, pero en el caso de los niños y/o jóvenes inmigrantes es en ella donde, además, aprenden y desarrollan el nuevo idioma (Suárez-Orozco y Suárez-Orozco, 2003). Así, la escuela se convierte en la principal institución encargada de llevar a cabo el proceso de asimilación lingüística, que, a su vez, constituye uno de los componentes fundamentales del proceso de asimilación cultural en EUA. Un país que se ha originado y ha crecido por la llegada de inmigrantes; que carece de una historia y de elementos comunes en los que fundar «el sentimiento de identidad nacional», por lo que la lengua se convierte en uno de los principales fundamentos del patriotismo estadounidense (Portes y Rumbaut, 2010).

\section{La escuela y el grupo de pares}

Los casos que se presentan a continuación son el de Isaac y Daniela, dos jóvenes nativos con algunas similitudes acerca de sus experiencias en el contexto escolar, en cuanto a sus relaciones con el grupo de pares.

La escuela como institución educativa, además de instruir en un conjunto de conocimientos científico-técnicos, lo hace también respecto a normas de comportamiento aceptables «socialmente». De ahí que se impongan sanciones contra aquellos cuyos comportamientos no se atienen a la norma, pero con consecuencias que, más que «normalizar», pueden contribuir a una mayor «desviación». Según han relatado varios informantes, algunas de las sanciones escolares más frecuentes ante conductas que no se 
adecuan a las expectativas escolares son: expulsión por un periodo de tiempo determinado; expulsión definitiva, después de varias previas; y/o derivación a otros centros. Muchas veces, este tipo de sanciones acaban comprometiendo el futuro de los jóvenes «problemáticos», ya que son derivados a centros específicamente orientados a este tipo de jóvenes, y cuyos programas se caracterizan por adaptaciones curriculares que se reflejan en un título final de menor calidad (para los que lo obtienen), el cual influirá en las futuras inserciones sociolaborales; o en el peor de los casos, muchos de estos jóvenes acaban teniendo problemas con la justicia e, incluso, pueden terminar en prisión.

Isaac, 16 años, generación 2.0

Nació en EUA y forma parte de una familia numerosa compuesta por tres hermanos, él y sus padres. Su hermana mayor y sus padres se encuentran indocumentados, mientras que él y sus dos hermanos tienen la ciudadanía por nacimiento. Reside en una de las ciudades de Orange con mayor tasa de población de origen latino y con menos recursos económicos. En unos meses comenzará su último curso de preparatoria.

-Eh, la cosa fue, este... que empecé muy chico a, a ser rebelde [...] empecé a hacer poquito, a no hacer mi tarea, a faltar a la escuela por equis razón. Y ya se va, se va empeorando la situación. Entonces, me veía expulsado de la escuela [...] y me metieron a escuelas del condado [...] entonces, ahi, pues desgraciadamente, hay gente rebelde: pandilleros, drogadictos. Y yo [...] tuve [...] meterme en ese [...] situación para $[\ldots]$ sentirme aceptado y para demostrar que yo no me dejo de nadie. Porque yo fue el único de ahi que me vestía así, normal, pero ya eso, viéndome, juntándome con los pandilleros, se empezó a cambiar mi modo de vestir, en mi estilo de pelo y todo.

Como relataba Isaac, su comportamiento fue el motivo principal de que lo mandaran a una escuela para jóvenes «problemáticos». Describía la nueva escuela como un centro totalmente diferente a los anteriores. La manera de trabajar era diferente, las tareas se realizaban mediante paquetes de trabajo y de manera individual, mientras el maestro estaba presente pero sin prestarles ninguna atención, y el horario era más corto.

Para él fueron varios los motivos por los que entró a la pandilla; el principal era que su nuevo grupo de amigos significaba un refugio ante la falta de atención por parte de su familia, sobre todo de su padre. Al mismo tiempo, constituía un orgullo contar con amigos que se apoyaban y se defendían mutuamente. Era algo de lo que podía presumir. Pero, finalmente, recapacitó sobre su situación y del daño que hacía a su madre, motivo 
por el cual decidió cambiar y convertir su trayectoria educativa en el objetivo central de su vida.

En una situación similar a la de Isaac se encontró Daniela, quien tras su llegada a California entró a formar parte de una pandilla.

Daniela, 26 años, generación 1.25-generación 2.0

De madre mexicana y padre austriaco, nació en EUA, pero vivió en Alemania hasta los catorce años, momento en el que su familia decidió volver a EUA. Su madre se encontraba indocumentada, pero regularizó su situación gracias al estatus de ciudadana de Daniela, después de que esta cumpliera los veintiún años. Actualmente se encuentra estudiando Administración de Negocios en un colegio comunitario.

-Yo tenía catorce años, estaba muy emocionada de haber llegado a los Estados Unidos [...] iba de diario a la alberca, la gente muy linda, la escuela a la que llegué muy buena. Me metí a ESL [...] o sea, yo no hablaba inglés cuando llegué a este país.

- ¿Y QUIÉNES ERAN TUS COMPANEEROS DE CLASE?

-Eran chicanos. O sea, puros mexicanos pero con no mucha educación [...] [...] también había como gente de Nigeria [...] muchos persas [...] turcos y así eran mis compañeros de ESL. Y ya salí de ESL y ya. Llegaron mis papás y me metieron a otra escuela pública y ahí fue cuando, como no hablaba inglés, me empecé a juntar, como, con los mexicanos. Y tristemente el área donde viviamos antes era un área no muy buena. Entonces, me empecé a juntar con ellos y pandillistas, y asi [...] era como que sentía que era el único grupo al que podía pertenecer, porque hablaban español [...] y luego una cosa que hacian aquí también: ;OH, SAY CAN YOU SEE BY THE DAWN'S EARLY LIGHT! [Canta el himno de los EUA] I AMN'T AMERICAN. I DON'T WANT TO PLAYER TO THE FLAG. Yo no me siento... «I AM A MEXICAN, I DON'T WANT TO PRAY A... Y me sacaron de la escuela tres días.

En el caso de Daniela, el desconocimiento del nuevo idioma y el área a la que llegó a vivir con sus padres, de un estrato socioeconómico bajo, son los principales motivos que la llevaron a entablar amistad con jóvenes de origen mexicano, ya que eran los únicos con los que se podía comunicar. Su pertenencia al grupo pudo ser la razón de su rebeldía, la cual demostraba mediante la inasistencia a algunas de sus clases o la negación a rendir homenaje a la bandera, lo que le supuso la expulsión de la escuela durante varios días. 
Por ello, antes de que la situación empeorara, sus padres decidieron enviarla a México con algunos familiares. Allí permaneció un año estudiando en una escuela privada. De esta manera, la separaron de su grupo de pares y tuvo la oportunidad de retomar su trayectoria educativa tras su regreso a EUA, donde cursó su último año de preparatoria e inició sus estudios en un colegio comunitario.

Sin embargo, Daniela relataba que su trayectoria educativa también se vio afectada, además de su paso por la pandilla, por el evento migratorio, el cual le supuso un doble retraso; el desconocimiento de la lengua y la peor calidad del sistema educativo público estadounidense respecto del alemán.

\section{Expectativas y aspiraciones adultas versus expectativas y aspiraciones juveniles}

Las expectativas y aspiraciones paternas son fundamentales, ya que han constituido una de las principales influencias en el inicio de las transiciones. Los casos que se presentan a continuación son los de Sandra y Claudio, dos jóvenes cuyos padres inculcaron y exigieron a sus hijos la continuación de las trayectorias educativas como única alternativa, lo cual puede tener consecuencias positivas, pero también negativas. Imbuir desde temprana edad el interés por el ámbito académico puede ayudar a partir de altas aspiraciones; pero, al mismo tiempo, los jóvenes pueden sentirse presionados por diferentes motivos, ya sea porque no comparten el mismo interés que sus padres en cuanto al ámbito educativo, prefiriendo optar por lo laboral; porque no comparten la opción educativa propuesta; o porque al llegar el momento todavía no han decidido, no tienen claro qué opción tomar. Por tanto, serán estos dos últimos supuestos a los que se hará referencia en los relatos.

\section{Sandra, 23 años, generación 1.5}

Llegó a los ocho años junto a su madre. Sus padres se separaron al poco tiempo de que ellas llegaran a EUA y desde entonces ha permanecido con su madre. Actualmente está finalizando sus estudios en el colegio comunitario y se prepara para entrar a la universidad.

-Entonces, yo estaba estudiando Negocios Internacionales cuando yo estaba en la preparatoria [...] yo pienso que ahorita, como mi generación, hay muchas personas que dicen: "quiero hacer esto», pero en realidad... Eso no es lo que a ellos les gusta estudiar. Nomás quieren porque sus papás los presionan a que: «quiero que seas un doctor», «quiero que seas un licenciado» [...] mi mamá siempre me decía que quería que estuviera en negocios internacionales [...] que es una carrera que tal vez me va ayudar en el futuro. 
Entonces, pues me enfoqué en eso [...] y nunca me gustó. Pero a mí siempre me gustó el ejercicio [...] me gustó mucho lo de nutrición [...] me empecé a informar, todo eso sobre: la nutrición [...] kinesióloga, lo que ahorita estoy estudiando. Y me gustó, me fascinó. Yo le comenté a mi mamá y le dije: "¿sabes qué?». Me senté con ella y yo le dije: «ma, este... yo no sé si te va a afectar un poquito. No sé. Tú siempre me decías que me metiera a negocios y, la verdad, lo hice, pero no me llamó la atención. Y ahora, yo puedo hacer algo que voy a trabajar y voy a ser feliz [...] ahorita lo que me estoy enfocando es, haciendo lo que a mi me gusta, que es kinesióloga [...] y hasta mi mamá [...] me está apoyando.

Sandra tomó la decisión de no seguir adelante con las aspiraciones de su madre, quien comprendió y aceptó su decisión gracias a la buena relación que ambas mantienen. Se trata de una relación basada en la comprensión, la confianza y el apoyo mutuo, en lugar de la autoridad que suele caracterizar las relaciones tradicionales entre padres e hijos. Además, al tratarse de una familia monoparental, la joven considera que el rol de su mamá ha sido doble, de madre y padre, por ello argumentaba que la mejor manera de devolverle todo lo que le había dado era trabajando duro y terminando sus estudios.

Piensa que los padres suelen obviar las aspiraciones de sus hijos, y en su lugar proyectan las propias. Finalmente, esto se convierte en el motivo por el que, muchas veces, los jóvenes no son felices, ya que no están a gusto con el trabajo que desempeñan, pues se dejaron llevar por los intereses de sus padres y olvidaron los propios. Los padres desean el bienestar económico y el prestigio social que asocian a algunas ocupaciones, pero no son conscientes de que hoy en día existen otras muchas profesiones que también pueden ofrecer un buen futuro.

En cambio, para Claudio, el caso que se tratará a continuación, sus estudios en Negocios Internacionales representa la opción con la que ha reconducido su trayectoria educativa después de haberla abandonado por un tiempo.

\section{Claudio, 24 años, generación 1.5-generación $2.0^{5}$}

Nació en EUA, pero vivió en México hasta los once años, momento en el que llegó a California para vivir con su abuela. Actualmente estudia Negocios Internacionales en la universidad.

${ }^{5}$ Claudio y Daniela nacieron en EUA, pero residieron fuera hasta los once y catorce años, respectivamente, momento en que regresaron. De ahí que sus experiencias sean equivalentes a las de las generaciones 1.5. y 1.25 , respectivamente. Sin embargo, no se puede obviar la pertenencia a la generación 2.0 por los derechos que concede el estatus de ciudadano. 
- ¿CUÁL ES EL MOTIVO POR EL QUE TÚ PASAS DIRECTAMENTE DE LA HIGH SCHOOL A LA UNIVERSIDAD?

- La expectativa y la presión que tenía de los profesores, como de mis padres [...] la educación en mi familia es algo que tienes que hacer. O sea, no tienes otra opción [...] mi último año [en la preparatoria] fue el más frustrante de toda mi vida [...] fue con mucho estrés [...] mi rendimiento no era bueno [...] solicité admisión a algunas universidades, pero no sabía muy bien qué quería estudiar, a qué universidad quería ir [...] estuve un año y medio en la universidad. Decidí darme un año de baja, precisamente, porque no sabia lo que quería estudiar. No sabía realmente qué es lo que quería hacer, cuál eran mis metas. Regresé a México. Ese año fue mi año sabático. Viví con mis papás [ríe], me lo pasé muy bien. Estuve trabajando con mis papás y al mismo tiempo estuve viajando alrededor de la República mexicana.

Deprimido por la situación en la que se encontraba y sin saber qué rumbo tomar, aceptó la propuesta de sus padres de marcharse un tiempo a México. Allí pasó un año, periodo en el que viajó, estuvo con su familia y trabajó. Todo ello le sirvió para reflexionar sobre lo que realmente quería hacer con su vida. Define el tiempo que pasó en México como «una etapa dura, de mucha responsabilidad y de aprender a manejar situaciones difíciles». Fueron experiencias que le ayudaron a forjar su carácter y a tomar una decisión sobre su futuro. Durante este periodo pensó en varias posibilidades; entre ellas barajó la posibilidad de quedarse en México y retomar sus estudios allí o aceptar la propuesta que le habían hecho sus padres de emprender negocios con ellos. Pero, finalmente, decidió que su futuro debía pasar por retomar su trayectoria educativa en EUA.

$\mathrm{Su}$ decisión final estaría muy influenciada por las experiencias de sus amigos, muchos de los cuales se estaban graduando de licenciatura y otros, incluso, estaban comenzando maestrías o trabajando para grandes corporaciones. Él también quería vivir esas experiencias, y las quería vivir en EUA, donde dispondría de ayuda financiera para poder continuar sus estudios. Por ello regresó y retomó sus estudios.

\section{Las hijas ante las expectativas y aspiraciones paternas}

Celina, 24 años, generación 2.0

Nació en EUA y es la menor de cuatro hermanos. Reside con sus padres en la ciudad donde nació y creció, la cual se caracteriza por contar con una gran proporción de población de origen mexicano y por carecer de servicios básicos de bienestar social, especialmente su área. Actualmente ha retomado sus estudios en el colegio comunitario. 
Durante la entrevista se refirió a un evento que podría haberse producido en su temprana juventud y que, de haberlo hecho, habría cambiado el transcurso de su transición a la adultez.

-Estaba en la iglesia en que conocí a un chavo y ya nos íbamos a casar. Y como, paré y dije: «NO, esto no es para mì. Like ¿Verdad? Me sacó de onda también. ¿QUÉ ES ESTO? ¿Realmente quiero tener hijos a los 20 años? ¿A los 18 años? Porque lo conocí, tenía 18 años, y porque dijeron ((los padres de ambos)): «se tienen que conocer dos años y se casan». ALL, ¿WHAT? A los 20 años no me voy a querer casar y no voy a tener hijos. ¡Fuck! ¡Not! [...] esos acuerdos, o la manera en que tiene que ser esta vida, a veces, no aplica a todos, y sería algo bien feo, bien triste, vivir por alguien más. Entonces, esa es la batalla de vivir por nuestros papás ¿Verdad? Por lo que ellos quieren que hagamos $[\ldots]$ yo hubiera tenido un niño [...] mi amiga tuvo a los 16, la otra tuvo a los 17, la otra tuvo a los 18. Y ahorita, cada una tiene tres niños [...] so, eso es muy común ¿Verdad? Pero también tiene que ver con [...] la religión, de la Iglesia Católica, que no quieren llegar al punto de decir: «somos seres humanos, somos muy estúpidos», a veces, y más cuando eres un chico, una chica que no sabes lo que estás haciendo.

Para Celina, convertirse en madre/padre tiene importantes implicaciones, sobre todo, cuando se produce a temprana edad, ya que los jóvenes se ven abocados a abandonar sus trayectorias educativas e iniciar las laborales en unas condiciones que no son las idóneas. Hacía referencia a algunas amigas, quienes tuvieron sus hijos a muy temprana edad, como ejemplo de lo que ocurre a muchas de las jóvenes de origen mexicano debido a las imposiciones familiares y a la influencia de la religión. De esta manera, los jóvenes inician sus transiciones a la vida adulta a través de un modelo que enmarca los itinerarios femeninos y masculinos en los roles de género tradicionales; el destino «natural» de las mujeres es convertirse en madre, mientras que el de los hombres es convertirse en el sustento económico principal. Así, la única salida posible y deseada para muchas mujeres es la formación de una nueva familia. Sin embargo, para Celina la maternidad no representaba, ni representa, una opción, al menos de momento, ya que, como argumentaba, no se sentiría bien «trayendo un hijo a un mundo tan dañado medioambientalmente». Por eso, los jóvenes y los adultos deberían pensar más seriamente acerca de las implicaciones de convertirse en madres/padres a tan temprana edad.

Defendía que los jóvenes deben conocerse más a sí mismos y adquirir la madurez suficiente para poder tomar decisiones importantes. Sin embargo, la mayoría de las familias asumen el modelo de transición clásico como el ideal de tránsito a la adultez, lo que significa, de nuevo, que los padres/madres imponen sus propias expectativas y aspiraciones sobre las de sus hijos. 


\section{La educación como base para los proyectos futuros y el papel del estatus legal}

Sofía y Fernanda son dos jóvenes que tienen muy clara sus aspiraciones educativas. Sofía está amparada por el Dream Act $^{6}$, mientras que Fernanda es ciudadana americana y ha vivido entre EUA y México. Ambas están estudiando en la universidad y en el colegio comunitario, respectivamente, motivos por los cuales no residen temporalmente con sus padres.

Sofía, 19 años, generación 1.75

Llegó con sus padres cuando apenas tenía dos años, situación por la cual se encuentra indocumentada. Actualmente está amparada por el Dream Act, lo que le ha otorgado, además de la seguridad de no ser deportada, el derecho a recibir ayuda económica para estudiar en la universidad.

- Estoy estudiando en la Universidad de California, quiero estudiar Ciencias Politicas [...] esta universidad era la que me ofrecía mejores becas, el mejor paquete completo. Me aceptaron en escuelas cerquita de aquí, pero tenía que estudiar y trabajar y preferí centrarme en estudiar [...] quería estar en una comunidad más chiquita. En un lugar más alejado de la ciudad para ver cómo era ese ambiente. Pero, también fue una decisión impulsiva, porque me quería ir. Quería estar lejos de mis padres para que yo también me enseñara. Cuidarme sola y que ellos tuvieran confianza que iba a estar bien [...] me enseñó mucho a estar sola, estar lejos de mis padres y tener responsabilidades.

Sofía ha optado por continuar su trayectoria educativa en una universidad que le ofrece un conjunto de becas que le permiten dedicarse exclusivamente a los estudios. Cuenta con dos becas dirigidas específicamente a estudiantes indocumentados, situación que ha aprovechado, ya que su estatus de indocumentada le restringe el derecho a aplicar a otras ayudas.

En su relato también hace referencia a la oportunidad de trasladarse a vivir a un nuevo lugar, alejada de sus padres. Esto le ofrece la posibilidad de aprender a vivir sola, de ser capaz de valerse por sí misma, así como de que sus padres aprendan a tener confianza en ella. En definitiva, el inicio de sus estudios universitarios le ha brindado nuevos retos y oportunidades, entre los que destaca el disfrute de mayores cotas de libertad.

${ }^{6}$ La propuesta de ley del Dream Act (Development, Relief and Education for Alien Minors) fue presentada en 2012 por el expresidente Barack Obama y daría lugar a la acción ejecutiva conocida como DACA (Deferred Action for Childhood Arrivals), cuyo objetivo no era conceder estatus legal, sino evitar la deportación y proporcionar autorización de empleo por un tiempo limitado. 
Como se verá en el caso que se expone a continuación, Fernanda también ha convertido su trayectoria educativa en un objetivo prioritario. Sin embargo, a diferencia de Sofía, su estatus de ciudadana estadounidense le ha permitido moverse entre México y EUA según sus intereses.

Fernanda, 20 años, generación 2.0

Nació en México, pero obtuvo la ciudadanía estadounidense a través de su padre, que es ciudadano estadounidense de origen mexicano. Ha vivido entre México y EUA.

-Yo estuve en el kínder allá [...] mi quinto y sexto año lo hice acá (EUA). Me quise regresar allá [a México] porque toda mi familia estaba allá [...] me quise venir acá porque pensé que era más fácil. Siempre trato de buscar mi camino más fácil para evitar problemas, y como no estaba pasando mis calificaciones allá, entonces, me vine para acá. Pasé y me gradué acá.

En un primer momento su plan era venir a California para estudiar y después volver a México para trabajar. Pero, una vez aquí, decidió que tras acabar sus estudios se quedaría. Ahora, su objetivo es continuar su trayectoria educativa en el colegio comunitario y después en la universidad con el fin de obtener su licenciatura en Comunicación y Negocios. Su meta es poder trabajar en EUA, ya que piensa que este país le puede ofrecer más calidad de vida que México.

Precisamente, con el objetivo de cambiar su situación económica actual retomó sus estudios en California. Sabe que un mayor nivel educativo le ayudará a encontrar un buen trabajo que le asegure mayores ingresos. Por eso aplica los diferentes beneficios sociales a los que puede acceder por su estatus de ciudadana. De ahí que el centro en el que estudia forme parte de una organización sin ánimo de lucro que le permite estudiar a muy bajo costo. Por otro lado, su estatus legal también le ha permitido moverse con facilidad entre ambos países, de manera que ha desarrollado su trayectoria educativa en función de sus propios intereses. Situación que se ha visto facilitada, y/o favorecida, por las redes familiares extensas con las que cuenta en ambos países.

\section{A MODO DE CONCLUSIÓN}

Como se ha evidenciado a través de los relatos de vida de los jóvenes, los aspectos que han influido en el inicio de sus transiciones a la adultez han estado en estrecha co- 
nexión con las relaciones de autoridad ejercida por los adultos. La escuela constituye el ámbito central de los principales intereses y conflictos en este momento del proceso de transición. Las expulsiones escolares de algunos de los jóvenes conlleva el cuestionamiento del rol de la institución educativa estadounidense ante las diferentes problemáticas juveniles. La expulsión es una opción que acaba excluyendo a los jóvenes del sistema educativo «normalizado», ya que son derivados a otros centros, destinados, específicamente, a niños y jóvenes categorizados como «problemáticos». De esta manera, también acaban siendo excluidos socialmente, ya que son expuestos a situaciones que facilitan la entrada en determinados grupos de pares, como las pandillas, que pueden constituir un refugio, pero también el preámbulo de la adhesión a una subcultura de la violencia. Los motivos por los que los informantes ingresan en las pandillas son diversos. Muchos de ellos son migrantes que llegaron a mayor edad, por lo que el desconocimiento del nuevo idioma les lleva a entablar amistad con pares de su mismo origen étnico. De esta manera, el grupo funciona como «grupo de referencia» en el que pueden sentirse integrados en los primeros momentos (Pais, 2007). En otros casos, los jóvenes simplemente buscan entre sus pares la atención que no encuentra en sus familias.

El aprendizaje del nuevo idioma también puede influir negativamente en el desarrollo global de la propia trayectoria educativa, especialmente en el momento en que se produce el paso de la preparatoria a los estudios superiores. Este tránsito también se ha revelado como una de las coyunturas claves para los jóvenes, ya que constituye el origen de los dilemas en torno a las trayectorias educativas y donde incide de manera importante la autoridad de los adultos. Entre las dificultades encontradas tras la finalización de la preparatoria destacan: las decisiones en torno a la continuidad o no de los estudios, qué estudiar, arrepentirse de las elecciones realizadas o la influencia de los padres/madres en sus decisiones.

El estatus legal de los informantes constituye un factor de peso. Los jóvenes que cuentan con ciudadanía y/o con permiso de residencia pueden acceder a ayudas y recursos financieros para los estudios, lo que constituye una gran ventaja. También posibilita la movilidad entre EUA y México, lo que contribuye a aumentar considerablemente las posibilidades de éxito educativo y laboral. Al mismo tiempo, las redes familiares transnacionales, y particularmente el apoyo de los familiares residentes en EUA, han resultado fundamentales para algunos informantes. Por otro lado, el amparo del Dream Act ha ofrecido alternativas a jóvenes indocumentados que de otra manera no habrían tenido debido a su estatus de indocumentados.

A pesar de las dificultades, los jóvenes de segunda generación de origen mexicano entrevistados para este trabajo cuentan con un mayor grado de autonomía en torno a sus proyectos de vida, los cuales se tornan más personales e individualizados. Proyectos 
vitales que difieren, en la mayoría de los casos, de las aspiraciones y expectativas paternas, pero sin que ello constituya un conflicto generacional, sino diferencias intergeneracionales que han supuesto la reestructuración de las relaciones paternofiliales. De esta manera, las relaciones entre padres e hijos se vuelven más igualitarias y se logran mayores niveles de comprensión y entendimiento mutuo, en sintonía con los múltiples cambios acaecidos en el seno de la familia nuclear (Meil, 2006; 2011).

\section{REFERENCIAS BIBLIOGRÁFICAS}

Aparicio, R. (2007). «Las «segundas generaciones» en España: marroquíes, dominicanos, peruanos». En Cuadernos Europeos de Deusto (36), págs. 19-56.

Beck, U. \& Beck-Gernsheim, E. (2003). La individualización. El individualismo institucionalizado y sus consecuencias sociales y políticas. Barcelona, España: Paidós.

Benedict, R. (2008). «Continuidades y discontinuidades en el condicionamiento cultural», en Pérez Islas, J. A. et al. (coords.): Teorías sobre la juventud. Las miradas de los clásicos. México: SES, UNAM; SIJ, UNAM; IISUE, UNAM; CIIJ; M.A. Porrúa.

Bengston, V. L. (2001). «Beyond the nuclear family. The increasing Importance of Multigeneracional Bonds». Journal of Marriage and Family (63), pags. 1-16.

Bertaux, D. (1999). «El enfoque biográfico: Su validez metodológica y sus potencialidades». En Proposiciones (29), págs. 1-23.

- (2005). Los relatos de vida. Perspectiva etnosociológica. Madrid, España: Ediciones Bellaterra.

Bourdieu, P. (2002). «La «juventud» no es más que una palabra», en Bourdieu, P. (comp.): Sociología y cultura. México, D.F., México: Grijalbo.

Cavalli, S. (2007). «Modèle de parcours de vie et individualisation». Gerontologie et societé (30-123), págs. 55-69.

Cavalli, A. \& Galland, O. (1995). Youth in Europe. London, U.K.: Pinter Pub Ltd.

Dávila, O. \& Ghiardo, F. (2011). «Trayectorias sociales juveniles. Cursos y discursos sobre la integración laboral». En Papers (96-4), págs. 1205-1233.

Eisenstadt, S. N. (2008). «Grupos de edades y estructura social», en Pérez Islas, J. A. et al. (coords.): Teorías sobre la juventud. Las miradas de los clásicos. México: SES, UNAM; SIJ, UNAM; IISUE, UNAM; CIIJ; M.A. Porrúa.

Epstein, A. L. (2006). «Etnicidad e identidad», en Camus, M.: Las ideas detrás de la etnicidad. Una selección de textos para el debate. Antigua, Guatemala: CIRMA, Centro de Investigaciones Regionales de Mesoamérica. 
Esping-Andersen, G. (2000). Fundamentos sociales de las economías postindustriales. Barcelona: Epaña, Ariel.

Feixa, C. (1998). De jóvenes, bandas y tribus. Antropología de la juventud. Barcelona, España: Ariel.

Galland, O. (1991). Sociologie de la jeunesse. París, Francia: Armand Collin.

Geertz, C. (1973). La interpretación de las culturas. Barcelona, España: Gedisa.

Gil Calvo, E. (2001). Nacidos para cambiar: cómo construimos nuestras biografias. Madrid, España: Taurus.

Giner, S. et al. (eds.) (1998). Diccionario de sociología. Madrid, España: Alianza.

Leccardi, C. (2010). La juventud, el cambio social y la familia: de una cultura de protección a una cultura de negociación. En Revista de Estudios de Juventud (90), págs. $33-42$.

Mayer, K. U. (2001). «The paradox of global social change and national path dependencies: life course patterns in advanced societies», en Woodward, A.E. y Kohli, M. (eds.): Inclusion and exclusion in European societies. Londres: Routledge.

Meil, G. (2006). Padres e hijos en la España actual. Colección de Estudios Sociales, 19. Barcelona, España: Obra Social «La Caixa».

- (2011). Individualización y solidaridad familiar. Colección de Estudios Sociales, 32. Barcelona, España: Obra Social «La Caixa».

Moreno Mínguez, A. (coord.) et al. (2012). La transición de los jóvenes a la vida adulta. Crisis económica y emancipación tardía. Colección de Estudios Sociales, 34. Barcelona, España: Obra Social «La Caixa».

Musgrove, F. (2008). «La invención del adolescente», en Pérez Islas, J. A. et al. (coords.): Teorías sobre la juventud. Las miradas de los clásicos. México: SES, UNAM; SIJ, UNAM; IISUE, UNAM; CIIJ; M.A. Porrúa.

Pais, J. M. (2007). Chollos, chapuzas, changas. Jóvenes, trabajo precario y futuro. Barcelona, España: Anthropos.

Parsons, T. (2008). «La edad y el sexo en la estructura social de Estados Unidos», en Pérez Islas, J. A. et al. (coords.): Teorías sobre la juventud. Las miradas de los clásicos. México: SES, UNAM; SIJ, UNAM; IISUE, UNAM; CIIJ; M.A. Porrúa.

Portes, A. \& Rumbaut, R. G. (2010). Legados. La historia de la segunda generación migrante. Barcelona, España: Hipatia.

Reguillo, R. (2012). Culturas Juveniles. Formas políticas del desencanto. Buenos Aires, Argentina: Siglo XXI.

Rumbaut, R. G. (2006). «Edades, etapas de la vida y cohortes generacionales: un análisis de las dos primeras generaciones de inmigrantes en Estados Unidos», en Portes, A. y DeWind, J. (coords.): Repensando las migraciones. Nuevas perspectivas teóri- 
cas y empíricas. Colección América Latina y el Nuevo Orden Mundial, México: Miguel Ángel Porrúa, UAZ, Secretaría de Gobernación Instituto Nacional de Migración.

Saraví, G. A. (2015). Juventudes Fragmentadas. Socialización, clase y cultura en la construcción de la desigualdad. México, D.F., México: CIESAS/FLACSO.

Singly, F. (2005). «Las formas de terminar y de no terminar la juventud». En Revistas Estudios de Juventud (71), págs. 111-121.

Suárez-Orozco, C. \& Suárez-Orozco, M. M. (2003). La infancia de la inmigración. Madrid, España: Morata.

Walter, A. (2004). «Dilemas de las políticas de transición: discrepancias entre las perspectivas de los jóvenes y de las instituciones». En Revista Estudios de Juventud (65), págs. 133-150. Madrid, España: Instituto de la Juventud. 